Supporting Information

\title{
X-ray Structure Analysis of Ozonides by the Crystalline Sponge Method
}

Shota Yoshioka, Yasuhide Inokuma, Vincent Duplan, Ritesh Dubey and Makoto Fujita*

Department of Applied Chemistry, School of Engineering, The University of Tokyo, 7-3-1 Hongo, Bunkyo-ku, Tokyo, 113-8656, Japan.

\section{Table of Contents}

S1. Experimental details

S2. Crystallographic analysis and crystallographic tables

S3. NMR Spectroscopy analysis

S4. References 


\section{S1. Experimental details}

Solvents and reagents were purchased from TCI Co., Ltd., WAKO Pure Chemical Industries Ltd., and Sigma-Aldrich and used without further purification. Ozone gas was generated by a SIBATA ozone generator. The concentration of ozone was measured by GASTEC GV-100 sampling device equipped with No. 18M detector tube. Crystalline sponge $\left[\left(\mathrm{ZnI}_{2}\right)_{3}(\mathrm{tpt})_{2}\right]_{n}(\mathbf{1}, \mathrm{tpt}=1,3,5$-tris $(4$-pyridyl)triazine $)$ was prepared according to the reported procedure. ${ }^{1}$ A screw-top microvial (Osaka chemical, cat. No. 11090620), screw cap with septum seal (Osaka chemical, cat. No. 53951-09FB) and a syringe needle (TERUMO, cat. No. NN-2116R) were used for the slow evaporation of the solvents during the guest inclusion.

\section{S2. Crystallographic analysis and crystallographic tables}

Single crystal X-ray diffraction data were collected on Rigaku XtaLAB P200 diffractometer equipped with rotating anode Mo-K $\alpha$ radiation source $(\lambda=0.71073 \AA)$ using a hybrid photon counting detector (DECTRIS Pilatus 200K) or SuperNova diffractometer (Rigaku Oxford) using micro-focus $\mathrm{Cu} K \alpha$ radiation $(\lambda=1.54184 \AA)$ equipped with $C C D$ detector. All data collection strategies were performed at $93 \mathrm{~K}$ using cold nitrogen stream. In all the reported crystal structures, data processing was performed with CrysAlisPro and the appropriate absorption correction (empirical and numerical) methods were applied.

Structure solution and refinement were performed using the SHELX $X^{2}$ program embedded in WinGX $X^{3}$ and Shelxle $e^{4}$ suits. All non-hydrogen atoms were refined anisotropically while hydrogen atoms were found at the calculated positions and were 
refined isotropically using the riding model. In the reported crystal structures, disordered guest and/or solvent molecules were treated with appropriate crystallographic restraints. Detailed structural analysis of all the crystal structures are as follows:

\section{X-ray Structure Analysis of $1 \cdot 3$}

The asymmetric unit of styrene ozonide contains three guest molecules with $37 \%, 42 \%$ and $82 \%$ partial occupancies. Here, one of the guest molecules occupies special position with 2-fold symmetry while one guest molecule shares its positional coordinate with solvent (cyclohexane) molecule. In our guest refinement strategy, we applied appropriate crystallographic restraints like SADI, RIGU, SIMU, SAME for the styrene ozonide guest molecules. We also utilized AFIX command for the guest molecule occupied at the crystallographic special positions in the unit cell. Solvent molecules with partial occupancies, diffused electron densities and large thermal motions were subjected to the suitable crystallographic restraints (DFIX, DANG, SIMU and ISOR) during the crystal structure refinement.

\section{X-ray Structure Analysis of $1 \cdot 5$}

Stilbene ozonide crystal structure contains two guest molecules, cis-5 and trans-5, with approximate $71 \%$ and $66 \%$, respectively. The trans-5 diasteromer shares its positional coordinates with one of the partially occupied solvent (cyclohexane) molecules. Because of partial occupancies and large thermal motions, guest molecules were subjected to the suitable crystallographic restraints (SADI, RIGU, SIMU and SAME) during our structure refinement strategies. Solvent molecules treated with appropriate 
crystallographic restraints like DFIX, DANG, SIMU and ISOR.

\section{X-ray Structure Analysis of $1 \bullet 6$}

Unlike $\mathbf{1 \cdot 5}$ (at $4^{\circ} \mathrm{C}$ ), stilbene ozonide (at $50^{\circ} \mathrm{C}$ ) contains four benzoic acid guest molecules in the asymmetric unit with $68 \%, 62 \%, 62 \%$ and $64 \%$ partial occupancies. We applied suitable crystallographic restraints/constraints (SADI, SIMU, RIGU, SAME and AFIX) in our refinement strategies to increase the data-to-parameter ratios and therefore to model large thermal motions of the guest molecules. Solvent molecules appropriately treated with restraints like DFIX, DANG, SIMU and ISOR.

\section{X-ray Structure Analysis of $1 \cdot 8$}

Among all crystal structures, cyclopentene ozonide crystal structure shows relatively poor thermal motions and diffused electron densities in the Fourier map. This is probably because of the highly reactive nature of ozonide that contains nearly 41 weight $\%$ of oxygen contents!! The asymmetric unit comprises of two guest molecules with $49 \%$ and $43 \%$ where one of the guest molecules occupy special positions in the unit cell. Here, guest molecules were treated with suitable crystallographic restraints SADI, RIGU, SIMU and SAME owing to there diffused electron densities and large thermal motions. Solvent ( $n$-hexane) molecules treated with restraints like DFIX, SIMU and ISOR. 
Table. X-ray experimental data for $\mathbf{1} \cdot \mathbf{3}, \mathbf{1} \cdot 5, \mathbf{1} \cdot \mathbf{6}$ and $\mathbf{1} \bullet \mathbf{8}$.

\begin{tabular}{|c|c|c|c|c|}
\hline & $1 \cdot 3$ & $1 \cdot 5$ & $1 \cdot 6$ & $1 \cdot 8$ \\
\hline CCDC No. & 1011447 & 1011448 & 1011449 & 1011446 \\
\hline Crystal system & Monoclinic & Monoclinic & Monoclinic & Monoclinic \\
\hline Space group & $C 2 / c$ & $C 2 / c$ & $C 2 / c$ & $C 2 / c$ \\
\hline$a(\AA)$ & $35.5334(9)$ & $35.0615(11)$ & $34.8492(12)$ & $34.440(2)$ \\
\hline$b(\AA)$ & $14.9227(3)$ & $14.9665(3)$ & $14.8584(5)$ & $14.9178(8)$ \\
\hline$c(\AA)$ & $30.6034(10)$ & $30.3099(7)$ & $31.0351(10)$ & $29.894(2)$ \\
\hline$\alpha\left(^{\circ}\right)$ & 90 & 90 & 90 & 90 \\
\hline$\beta\left({ }^{\circ}\right)$ & $102.176(3)$ & $101.487(2)$ & $101.858(3)$ & $99.307(6)$ \\
\hline$\gamma\left({ }^{\circ}\right)$ & 90 & 90 & 90 & 90 \\
\hline$V\left(\AA^{3}\right)$ & $15862.5(8)$ & $15586.5(7)$ & $15727.1(9)$ & $15156.4(16)$ \\
\hline $\mathbf{Z}$ & 8 & 8 & 8 & 8 \\
\hline Density $\left(\mathrm{g} / \mathrm{cm}^{3}\right)$ & 1.790 & 1.745 & 1.668 & 1.628 \\
\hline Temperature (K) & 93 & 93 & 93 & 93 \\
\hline$\mu\left(\mathrm{mm}^{-1}\right)$ & 19.833 & 3.354 & 19.938 & 20.622 \\
\hline $\mathbf{F}(000)$ & 8278 & 7868 & 7536 & 7098 \\
\hline Crystal size & $0.42 \times 0.15 \times 0.13$ & $0.23 \times 0.13 \times 0.05$ & $0.30 \times 0.18 \times 0.11$ & $0.47 \times 0.17 \times 0.12$ \\
\hline Total reflections & 33769 & 34821 & 32570 & 27681 \\
\hline Unique reflections & 13993 & 13432 & 15525 & 13377 \\
\hline $\boldsymbol{R}_{\text {int }}$ & 0.045 & 0.032 & 0.032 & 0.046 \\
\hline Completeness (\%) & 100 & 97.6 & 97.0 & 99.8 \\
\hline GoF & 1.02 & 1.10 & 1.08 & 1.06 \\
\hline $\mathbf{R}_{1}\left(\mathbf{F}^{2}\right)$ & 0.0633 & 0.0553 & 0.0699 & 0.0949 \\
\hline $\mathrm{wR}_{2}\left(\mathbf{F}^{2}\right)$ & 0.1893 & 0.1719 & 0.2177 & 0.2978 \\
\hline
\end{tabular}




\section{S3. NMR Spectroscopy analysis}

${ }^{1} \mathrm{H}$ NMR spectra were recorded on a JEOL $500 \mathrm{MHz}$ spectrometer equipped with RO5AT/FGSQ probe. NMR spectra data were recorded at $300 \mathrm{~K}$ and chemical shift values are reported in parts per million (ppm) relative to an internal standard tetramethylsilane (TMS)
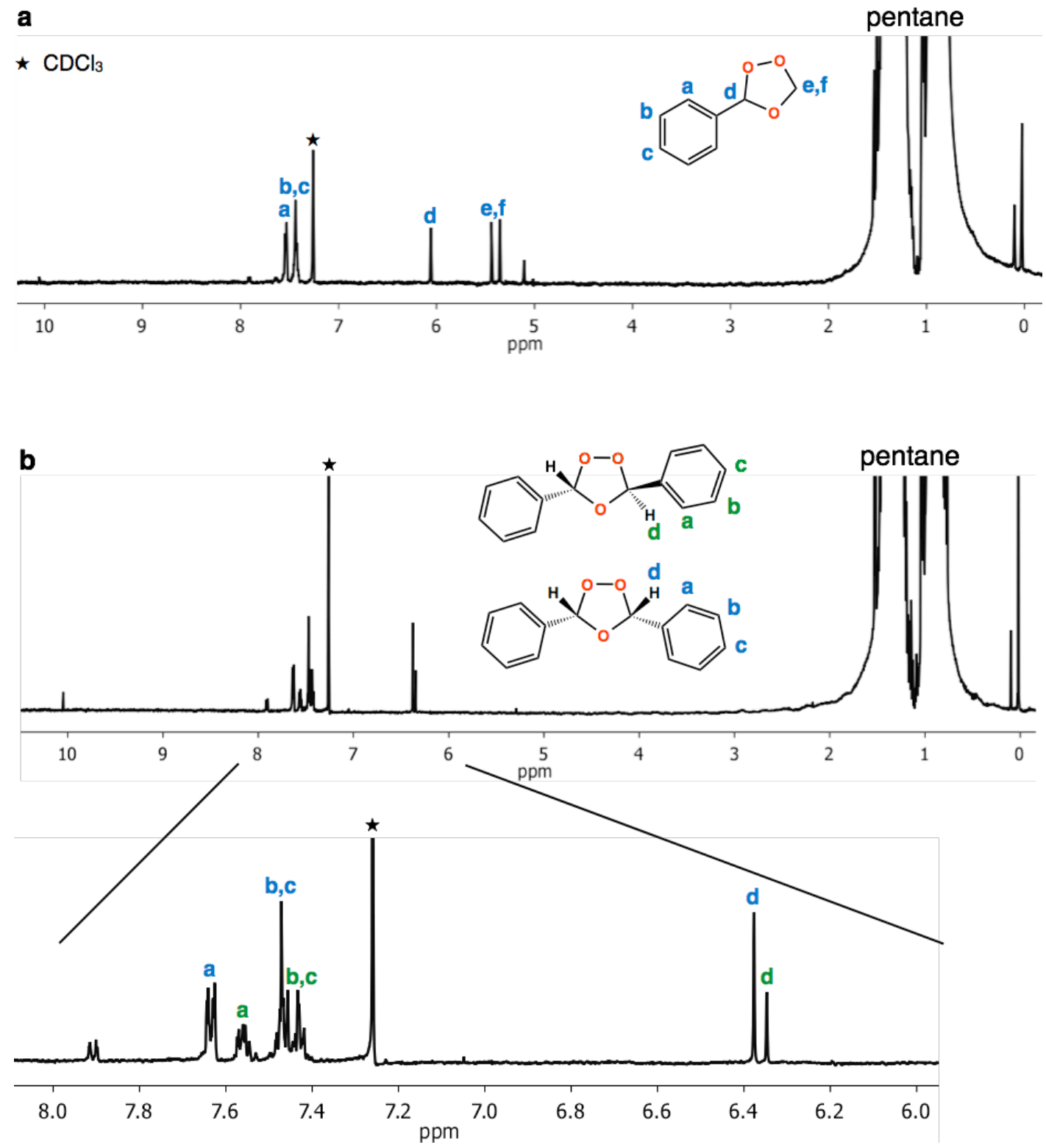
C

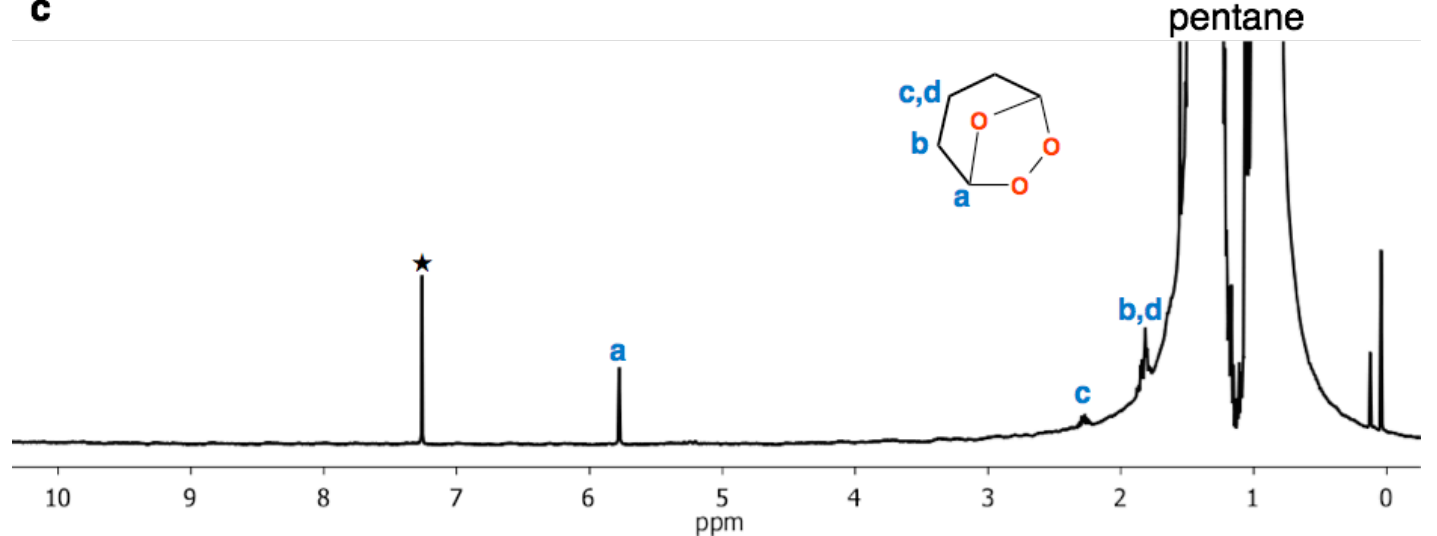

${ }^{1} \mathrm{H}$ NMR spectra $\left(500 \mathrm{MHz}, \mathrm{CDCl}_{3}, 300 \mathrm{~K}\right)$ of the reaction mixtures used for the direct guest inclusion of ozonides. (a) ozonide 3 from ozonolysis of styrene, (b) ozonide 5 (cis/trans mixture) from cis-stilbene, and (c) ozonide 8 from cyclopentene.

\section{S4. References}

1. Inokuma, Y.; Yoshioka, S.; Ariyoshi, J.; Arai, T.; Fujita, M. Nat. Protoc. 2014, 9, 246-252.

2. Sheldrick, G. M. Acta Cryst. 2008, A64, 112-122.

3. Farrugia, L. J. J. Appl. Cryst. 2012, 45, 849-854.

4. Hübschle, C. B.; Sheldrick, G. M.; Dittrich, B. J. Appl. Cryst. 2011, 44, 1281-1284. 